\title{
El difícil proceso de configuración de las arenas políticas nacionales en El Salvador y Nicaragua. El impacto de la guerra y la crisis
}

\author{
Ana Sofia Cardenal \\ Salvador Martí \\ Universitat Autònoma de Barcelona. Departamenc de Ciència Política i de Dret Públic. \\ 08193 Bellaterra (Barcelona). Spain
}

\section{Resumen}

Este artículo se centra en la evolución política de El Salvador y Nicaragua. En la primera parte se realiza un breve resumen de la historia política de ambos países hasta el estailido de la crisis revolucionaria a finales de los años setenta. En la segunda parte se abordan las consecuencias políticas de la guerra y se discute cómo ésta (la guerra) consiguió moldear las arenas políticas que emergieron en estos paises al finalizar el conflicto.

Palabras clave: guerra, transiciones democráticas, El Salvador, Nicaragua.

Abstract. The difficult process of shaping national political arenas in EI Salvador or Nicaragua. The impact of war and the crisis

The political evolution of Nicaragua and El Salvador is the main object of this article. The first section makes a brief summary of the political history of both countries up to the revolutionary crisis of the late seventies. The second section addresses the political consequences of the war and discusses how it has shaped the configuracion of the political arenas that emerged in these countries once the war was finished.

Key words: war, democratic transitions, El Salvador, Nicaragua.

\section{Sumario}

Las consecuencias políticas de la guerra El Salvador: empatc militar, derrota política

Nicaragua: la dificil cristalización

A modo de conclusión

de una arena politica Bibliografía

A finales de los años setenta los países centroamericanos (a excepción de Costa Rical) entraron en una profunda crisis de legitimidad (Booth, 1991; LaFeber, 1983; Torres-Rivas, 1983). Dicho fenómeno coincidió cronológicamente con

1. El desartollo político de Costa Rica, norablemente diferente del existente en el resco de los paises del istmo, tomará un camino completamente divergente a partir de la guetra civil acaccida en 1948. 
el súbito colapso del modelo de Estado "nacional-popular" en la mayor parte de países de Suramérica (la crisis de la deuda, la crisis fiscal del Estado, la inadecuación de sus mecanismos de articulación política) y con la creciente erosión de los regímenes autoritarios existentes en la mayor parte del subcontinente. Los acontecimientos en El Salvador y Nicaragua, sin embargo, responderán a la especificidad de sus arenas políticas.

Si bien las transiciones hacia regímenes civiles en buena parte de países suramericanos y la eclosión de la crisis política en el istmo coincidieron en el world time, la naturaleza de los actores, las coaliciones entre éstos, los métodos utilizados y sus objetivos fueron totalmente diferentes.

El carácter hermético de los regímenes nicaragüense y salvadoreño y su rechazo a cualquier pretensión reformista produjo la confluencia de buena parte de los movimientos opositores hacia la canalización de su actividad fuera de los canales institucionales que ofrecía el régimen. Éstos, que finalmente confluyeron con organizaciones de carácter político-militar, protagonizaron un importante rol en la crisis, y posterior quicbra, del modelo de Estado oligárquico. A la vez, la diferente configuración entre los dos regímenes fue una variable clave para la comprensión del triunfo revolucionario en Nicaragua y la imposibilidad de la victoria de la convocatoria insurgente propugnada por FMLN en El Salvador (Wickham-Crowley, 1992). El desarrollo, la complejidad y el cariz de estos procesos no será ajeno a la siempre omnipresente intervención de las diferentes administraciones norteamericanas (America's Warch; LaFeber, 1983; Schoultz, 1981; Pastor, 1987).

\section{Las consecuencias políticas de la guerra}

La ley marcial continuaba, en previsión de que fucra necesatio aplicar medidas de emergencia para la calamidad pública del aguacero interminable, pero la tropa estaba acuartelada. Durante el día los militares andaban por los torrentes de las calles, con los pantalones enrollados a media pierna, jugando a los naufragios con los niños. En la noche, después del toque de queda, derribaban puertas a culatazos, sacaban a los sospechosos de sus camas y se los lievaban a un viaje sin regreso. Era todavía la búsqueda del exterminio de los malhechores, asesinos, incendiarios y revoltosos del Decreto Número Cuatro, pero los militares lo negaban a los propios parientes de sus vícrimas, que desbordaban la oficina de los comandantes en busca de noticias. «Seguro que fue un sueñon, insistían los oficiales. "En Macondo no ha pasado nada, ni está pasando ni pasará nunca. Este es un pueblo felizm.

Gabriel García Márquez, Cien años de soledad.

Al intentar analizar los efectos políticos de diez años de guerra, agresión y conflictos en El Salvador y Nicaragua cabe citar, en primer lugar, la ya conocida frase de Karl von Clausewitz «la guerra es la continuación de la política por otros medios». Según el attor citado "la guerra es un acto de fuerza para imponer nuestra voluntad al adversario". 
Es precisamente desde esta óptica en la que cabe observar la contundente intervención de los EE UU en Centroamérica durante la década de los ochenta. Sin embargo, ningún análisis de las relaciones entre EEUU y Centroamérica puede prescindir de que dicha potencia ha significado un factor decisivo en el desarrollo de la región y que su influencia ha sido permanente, a la vez que la actividad exterior del gran vecino del norte ha estado históricamente regida por dos directrices: la consciencia de desarrollar una misión histórica -noción directamente imbricada con la doctrina del destino manifiesto-, y la creencia de que sus intereses vitales también se definen en el exterior, ampliando, de esta forma, la definición y los contenidos de su «seguridad nacionalm. Ambos principios apenas han experimentado variaciones en el tiempo (Aguilera y otros, 1991).

En el presente siglo los EE UU pasaron de la política del big stick, durante las primeras tres décadas, a la del good neighbor, bajo Franklin D. Roosevelt y Harry Truman. Luego siguieron políticas directamente intervencionistas en el marco de la guerra fría, bajo la administración de Dwight Eisenhower hasta la de Gerald Ford. Posteriormente, con la presidencia de Jimmy Carter, se intentó llevar a cabo una tenue política de promoción de derechos humanos que fue revertida por los acontecimientos y por la siguiente administración republicana de Ronald Reagan, que revitalizó la doctrina Monroe. A través de casi noventa años, sin embargo, la prioridad de las diferentes administraciones estadounidenses ha sido mantener el control en su "patio trasero". A lo largo del tiempo, bajo diferentes retóricas y estilos, el eje central de la política nortcamericana no ha cambiado: el mantenimiento del control y la estabilidad en una zona que siempre ha concebido como propia.

Los EEUU percibieron el triunfo de la revolución sandinista no sólo como una amarga derrota a su política exterior — pues Somoza no era un simple aliado sino su valedor y más estable interlocutor en la región-- sino una amenaza real a sus intereses geoestratégicos en la zona (Di Palma \& Whitehead, 1986). Desde esta óptica, la Administración republicana de Reagan enfatizó el conflicto centroamericano como el eje clave de su política exterior. Los discursos de Reagan, Kirkpatrick, Enders o Haig fueron gráficas expresiones de la centralidad del proceso político del istmo en el desarrollo de la política norteamericana (I.aFeber, 1993: 275-28I).

Siguiendo a Carlos Sojo, son tres las dimensiones que permiten resumir la naturaleza de la intervención de los EE UU en Centroamérica durante los años ochenta. La primera es la dimensión "contrarrevolucionaria", donde se argumentaba que toda transformación social en su área de influencia sería interpretada como acercamiento a la potencia enemiga y, en consecuencia, tenía que ser no sólo combatida y contenida sino también recuperada. Se trataba del llamado roll back - de la recuperación de espacios perdidos en el tablero de una confrontación bipolar-, el escenario de dicha polírica iba a ser Nicaragua. La segunda dimensión es la "contrainsurgente", en la cual se buscaba evitar la pérdida de otros espacios combatiendo y aislando a los actores que propugnaban la transformación del orden existente, siendo sus espacios de aplicación 
El Salvador y Guatemala. Finalmente, la tercera dimensión, llamada "de acción preventiva», trataba de preservar y consolidar sus actores aliados para evitar la creación de condiciones favorables a la emergencia de movimientos de naturaleza insurgente -el establecimiento de proyectos comerciales como la Iniciativa para la Cuenca del Caribe o el generoso flujo de créditos en aras de consolidar y unificar un sector privado y moderno son un gráfico ejemplo de ello-. Las tres dimensiones expuestas se articularon doctrinariamente en el documento "sobre la nueva política interamericana para los años ochenta" -conocido mayoritariamente como "documento de Santa Fe In-, y se ejecutaron bajo la estrategia de la llamada Guerra de Baja Intensidad (Barry y otros, 1987; Núñez y otros, 1991).

Sin duda alguna, el primer éxiro de la política Reagan fue extender la percepción de que el conflicto centroamericano respondía a la lógica bipolar EsteOeste en una realidad donde, tal como expuso un observador privilegiado (Ambler Moss, embajador de los EE UU en Panamá en 1980), «lo que estamos observando en América Central no sería muy diferente si no existiera Cuba ni la URSS".

Casi quince años después del inicio del estallido de dicha crisis es difícil hacer un balance sobre los costes que supusieron la violencia política y la confrontación social, prueba de ello es la dificultad de hallar cifras fiables sobre los efectos directos de la guerra y la complejidad de establecer unos criterios precisos a la hora de valorar el alcance de los efectos indirectos. Con todo, son muchos los estudios que han evaluado, medido y cuantificado en términos económicos y humanos los efectos de tal conflagración. En esta segunda parte del trabajo, sin embargo, pretenderemos esbozar alguna hipótesis sobre las consecuencias de la agresión norteamericana en la articulación de los sistemas políticos de El Salvador y Nicaragua.

\section{Nicaragua: la difícil cristalización de una arena política}

El proyecto contrarrevolucionario se gestó en el mismo momento en que las autoridades revolucionarias reorganizaron los poderes del Estado, en el mismo momento en que los sectores desplazados del poder observaron que perdiendo el control de los tesortes institucionales y del monopolio de la fuerza se disolvía la gravitación de sus intereses.

Para poner en marcha el movimiento contrarrevolucionario los sectores más duros de la derecha nicaragüense y de los EE UU diseñaron un proyecto dirigido a deslegitimar el nuevo proceso político. Como bien definió uno de sus máximos dirigentes, el fenómeno contrarrevolucionario se convirtió inicialmente en un proyecto donde "los argentinos pondrían los asesores militares; los hondureños, el territorio; los norteamericanos, el dinero, y los nicaragüenses la gente (Cruz en Núnez y otros, 1991: 143). La política contrarrevolucionaria nació en Managua y Washington, se organizó y se preparó en Centroamérica, se financió en los EE UU y se llevó a cabo en las árcas rurales nicaragüenses. 
La contra tuvo como objetivo, durante la mayor parte de su existencia -hasta 1986, fecha en que estalló el affaire Irán-Contra-, el derrocamiento militar del régimen de Managua a partir de dos premisas: 1) la creación de una estructura político-milirar alternativa capaz de vencer en el campo militar, de manera progresiva pero sistemática, al sistema político fruto de la revolución, y 2) el desgaste de las fuerzas armadas sandinistas y la progresiva deslegitimación popular del gobierno.

Al principio la organización contrarrevolucionaria se constituyó en base al bloque somocista desplazado del poder. Posteriormente, fruto de presiones exteriores, de desavenencias con la dirección sandinista y de errores en la ejecución de determinadas políticas por parte del gobierno, diferentes cuadros políticos y ciertos colectivos - son ejemplo de elio las comunidades misquitas de la Costa Arlántica o los pequeños y medianos propietarios rurales del norte $\mathrm{e}$ interior-- se fueron uniendo al grueso de las fuerzas contrainsurgentes (Bendaña, 1990; González, 1991; Christian, 1986; Orlando y otros, 1991; Robinson \& Norsworthy, 1987).

Durante el primer período la agresión de la contra se caracterizó por la progresiva organización de una guerra de baja intensidad articulada a partir de los medios económicos y las directrices emanadas de las agencias contrainsurgentes norteamericanas (lbarra, 1991; LaFeber, 1993). Entre 1985 y 1987 se dirimió buena parte del conflicto. En ese período el sandinismo mostró su preeminencia y solidez en la arena militar, obligando a la contra a replegarse y convertirse en un elemento de desgaste - y no una fuerza capaz de derrocar al régimen revolucionario por la vía bélica-. Las claves de este giro en la evolución de la guerra fueron cinco: 1) la disminución de la legitimidad y el apoyo de las bases campesinas hacia la contra a partir de los reajustes de la política agraria sandinista llevados a cabo en 1985 (Bendaña, 1991; Núñez y otros, 1991; Vilas, 1991a); 2) la práctica disolución del frente contrainsurgente del sur en 1986; 3) el cambio de equilibrio en la correlación de efectivos militares a favor del Ejército Popular Sandinista; 4) el estallido del escándalo IránContra que comprometía a las más altas jerarquías del ejecutivo estadounidense en una guerra sucia contra Nicaragua, y 5) el establecimiento, por primera vez, de un diálogo entre los cinco presidentes de la región con el objetivo de solucionar los conflictos del istmo por la vía del diálogo y sin injerencias exreriores --se trata, evidentemente, del acuerdo de Esquipulas II- (Aguilera y otros, 1991; Tinoco, 1989).

Con el fracaso de la estrategia guerrerista, las opciones en la lucha contra el gobierno sandinista quedaron reducidas a dos: 1) finalizar la guerra con una rápida y contundente victoria en el plano militar, hecho que suponía la intervención directa de las fuerzas norceamericanas, y 2) establecer una agresión prolongada con el objetivo de presionar y desgastar al régimen sandinista, a la vez que se desplazaba la gravedad de la ofensiva al plano de la oposición civil con el objetivo de forzar acuerdos que desgastaran progresivamente a los sandinistas; pues, aunque el gobierno de Managua tenía capacidad para continuar técnicamente la guerra, ésta le exigía buscar otras vías para su finalización, 
ya que los costos que suponía conllevaban una galopante erosión del capital político conseguido en la revolución (Martí, 1994b).

Así pues, la contra, con diversos nombres y configuración orgánica (de las diferentes siglas y grupos existentes en su inicio se dio paso a la Unión Nacional Opositora en 1985, para refundarse posteriormente, en mayo de 1987, bajo el nombre de Resistencia Nicaragüense), mantuvo actividades de sabotaje y agresión al proyecto revolucionario hasta marzo de 1990. Cabe observar, sin embargo, que la contra no fue un movimiento guerrillero más, sino que llegó a constituir un ejército especial enclavado en Nicaragua por los norteamericanos (de la misma forma en que lo fucron en el ámbito económico las plantaciones bananeras). La contra a finales de la década de los ochenta era un ejército con vastos recursos materiales, una retaguardia, logistica sofisticada, artillería, medios aéreos de transporte, aparatos nacionales e internacionales $\mathrm{y}$. un buen número más de objetos y pertrechos bélicos afincados en Honduras (Núnez y otros, 1991: 170-180).

La orquestación de una agresión bélica de semejante tamaño supuso que al "complejo" e "inestable" proyecto político iniciado por los sandinistas se sumara el factor "hostilidad". De las tres variables la hostilidad pronto se apropib del contexto y, con ella, emergieron dinámicas que reforzaron la centralización, la verticalización y la cohesión orgánica del régimen y del FSLN.

Con el inicio de la agresión se descompuso la llamada "luna de miel» con los sectores moderados que, junto con los sandinistas, confeccionaban la coalición de Unidad Nacional. Con el ascenso de la escalada belicista se redefinieron los vértices y las prioridades de la revolución. La estrategia norteamericana era clara -y también clásica - ísta puede ilustrarse gráficamente con la cita de Clausewitz: «si la victoria rápida del enemigo no es posible, se tiene que concentrar todos los esfuerzos para desgastarlo: incrementarle su coste de la guerra, destruir su territorio, aumentar su sufrimiento, y desgastarlo poco a poco, debilitando su posición moral y física[...] Se tiene que eliminar su voluntad de resistencia a través de operaciones que tengan repercusiones políticas directas". Y, evidentemente, la agresión comportó consecuencias políticas (Drago, 1988).

A partir de 1982 la revolución ya no podía seguir su marcha sin tener en cuenta los problemas gencrados por la guerra. Se articularon todos los instrumentos del poder en torno a un objerivo: la defensa. Tal como expuso el sociólogo nicaragüense Luís Serra unna limitante explicativa fundamental del avance del proceso de democratización política lo constituyó el fenómeno de la guerra. Una situación bélica prolongada adquiere una lógica propia que implica la estructuración particular de las relaciones saciales y del mundo simbólico. Las fuerzas armadas se expanden rápidamente, esto significa el enctuadramiento de gran parre de la población en una estructura militar caracterizada por su naturaleza jerárquica” (Serra, 1988). La guerra generó exigencias y creó condiciones para el fortalecimiento del control, la centralización y el verticalismo. Eficacia, celeridad y disciplina fueron las consignas de la nucva etapa. Se adoptó la doctrina de que la contra no tenía otro origen ni otros intereses que los de 
la Administración norteamericana - que era un enemigo exterior y que obedecía a causas y factores externos-, lo cual hacía muy difícil aceptar la existencia de otras causas relacionadas con el desarrollo efectivo del proceso político revolucionario - hecho que suponía una menor capacidad de acción y rectificación del régimen-- (Vilas, 1991a).

A finales de la década de los ochenta el proyecto sandinista estaba prácticamente exhausto: la economía estaba colapsada, el estrangulamiento económico de la Administración de los EE UU persistía y la ayuda del bloque del Este disminuía velozmente. Se negociaba el desenlace de una sociedad cansada y polarizada entre quienes lucharon en el proyecto revolucionario y quienes observaban la rápida esclerotización de éste (Torres-Rivas, 1990).

De los acuerdos iniciados en Esquipulas II el régimen político nicaragüense salía claramente reforzado. Éstos supusieron la legitimación internacional de las instituciones, de la Constitución y del proyecro político gestado en el marco de la revolución. Desde esta perspectiva, la estrategia contrarrevolucionaria de la reversión (o roll back) diseñada por la Administración republicana de los EE UU fracasó. El sistema político no sólo sobrevivió a la ofensiva militar, sino que también se perpetuaba y obtenía una legitimidad incuestionada.

Con todo, la pertinaz y contundente política de agresión afectó de lleno en el desarrollo de la articulación política del modelo emergente. Además de los costes en términos contables (LaFeber, 1993: 362-366) la política de "acoso y derribo" patrocinado por Reagan tuvo dos consecuencias vitales:

1. Significó la rápida disolución de la amplia coalición política que emergió al inicio de la década de los ochenta y el progresivo posicionamiento de los sectores moderados fuera de los cauces institucionales que ofrecía el nuevo marco político. Sin duda, el rápido desplazamiento de los actores opositores hacia posturas antisistema supuso el sistemático cuestionamiento de los espacios políticos que ofreció la revolución, así como la imposibilidad de articular eficazmente las demandas políticas de los diferentes colectivos.

2. La dinámica bélica, con sus costes económicos, organizacionales, y su efecto polarizador, supuso la imposibilidad de llevar a cabo en Nicaragua, por primera vez, la creación de un Estado nacional-popular y, por lo tanto, la integración de las masas populares en la comunidad política. Como ya hemos expuesto al inicio del trabajo, el complejo proceso de construcción de un Estado integrador en una época en que en el resto del subcontinente dicho modelo estaba en plena crisis y en que el marco internacional dificultaba su consolidación, el peso de la agresión patrocinada por la Administración norteamericana supuso el tiro de gracia a su ya difícil cristalización.

La situación política actual, caracterizada por una frágil estabilidad y por súbitos enfrentamientos que ponen en tensión los débiles resortes en que se sostiene el sistema, no es, en ningún caso, ajena a una década de constante acoso, violencia y destrucción. 


\section{El Salvador: empate militar, derrota política}

Tal como expone Cardenal en su trabajo, en 1979 se produce un punto de inflexión en la realidad política de El Salvador; el golpe milirar de octubre pone frn a una etapa caracterizada por una sólida alianza entre la oligarquía y las fuerzas armadas y por la exclusión y subordinación de grandes colectivos por parte del régimen (Cardenal, 1993). Dicho golpe fue producto de las divisiones dentro del seno del bloque dominante, agravadas y acentuadas por el auge de un poderoso movimiento popular que se había ido gestando desde inicios de la década de los setenta (Dunkerley, 1982).

Los objetivos de los sectores reformistas, artífices del golpe de octubre, pronto se vieron frustrados. La autonomía de los cuerpos armados salvadoreños, la presión y movilización ejercida por los movimientos populares y la vacilante actitud de la Administración Carter frente a los acontecimientos - temerosa ante la repetición de lo sucedido en Nicaragua - fueron las claves del fracaso reformista y los ingredientes para la posterior polarización y enfrentamiento. $\mathrm{L}_{a}$ teorganización de las diferentes organizaciones políticas - FMLN-FDR, por un lado, y PDC, FFAA y la oligarquía, por otro- en dos bloques diferenciados y antagónicos dieron como resultado el comienzo de una guerra.

En este marco, los EE UU pronto ejercieron un rol de gran importancia en el desarrollo de los acontecimientos. La lectura Este-Oeste en que se observó el conflicto por parte de la Administración Reagan hizo que los acontecimientos políticos de este pequeño país tuvieran una gran relevancia para el vecino del norte - El Salvador, en los años ochenta, pasó a ser el tercer país con mayor asistencia económica y militar de los EEUU, después de Israel y Egipto-(Gettleman y otros, 1981).

Entre 1980 y 1982 se desarrolló el período de implantación de la guerrilia, pero también el fracaso de sus planes iniciales. La ofensiva general desatada en 1981 no logró los objetivos militares, a la vez que en el campo civil el movimiento popular --tras un primer exito en cuanto a su capacidad de movilización- entró en una fase de agotamiento, fruto de la contundente represión - -durante ese bienio perdieron la vida 60.000 salvadoreños - (Ibarra, 1991). Simultáneamente, en el otro lado del espectro político, se consolidó el proyecto «reformista" contrainsurgente articulado por la Democracia Cristiana y apoyado y financiado por los EE UU.

La estrategia contrainsurgente tuvo desde sus inicios dos vertientes básicas: una militar y una política. La estrategia militar se reformuló con el tiempo por la necesidad de adaptarse a la naturaleza cambiante de la guerra. En un primer momento ésta obedeció a concepciones militares clásicas -concentración de tropas, grandes campañas, etc.- sin embargo, el resultado fue negativo para el ejército y, a partir de 1984, conscientes de que no se podía acabar con la guerrilla de forma rápida, se articularon nuevas medidas en el marco de la estrategia una "guerra de baja intensidad" con el objetivo de minar los apoyos de la guerrilla, cortarle las fuentes de provisión y reducir su capacidad de resistir una larga contienda. 
A nivel político, las directrices de la estrategia contrainsurgente se mantuvieron a lo largo de todo el conflicto. En su diseño figuraban tres actores centrales: Ios EE UU, el Partido de la Democracia Cristiana y las fuerzas armadas (Karl, 1986; Karl, 1989). Con el tiempo, sin embargo, un nuevo partido que aglutinó a la oligarquía salvadoreña - ARENA - adquiría gran preeminencia -en detrimento del PDC-, replanteando las alianzas anteriores y los posibles desenlaces. A pesar de la recomposición de las coaliciones, la mutación interna de los diferentes actores y su progresivo cambio en la percepción de las posibles salidas al conflicto armado, la estrategia política contrainsurgente para hacer frente a la propuesta revolucionaria propugnada por el FMLN/FDR fue, en gran medida, eficaz.

Con el tiempo, la creación de una institucionalidad alternativa a la propuesta lanzada por la insurgencia fue ganando solidez. La transición hacia gobiernos civiles - aunque dirigida desde fuera y desde arriba - tuvo la virtud de articular una nueva institucionalidad sobre la cual los EE UU reconoció un gobierno democrático y electo con el cual cooperar abiertamente. Si bien buena parte de los estudiosos de la política latinoamericana calificaron al régimen salvadoreńo como una "democracia de fachada" (Solórzano, 1983) o una "dernocracia ficticia" (Seligson, 1987; Berntzen, 1993), éste ejerció de forma efectiva su función de aislar cualquier otro modelo alternativo de articulación política.

A pesar de las primeras propuestas de diálogo lanzadas por el FMLN en 1982 y 1984, éste creyó en el desenlace militar hasta 1989. El desarrollo de los acontecimientos a partir de 1981 —debido a la peculiar articulacion de las fuerzas políticas y socioeconómicas salvadoreñas - dieron como resultado una larga guerra caracterizada por su carácter de clase (Wicham-Crowley, 1993: 282-288). El empantanamiento del conflicto y la imposibilidad de vislumbrar ningún desenlace bélico a corto plazo iba en detrimento de la convocatoria insurgente.

A finales de la década, después de diez años de guerra y esfuerzos, el cansancio era el lugar común de los contendientes. La imposibilidad de una rápida victoria por ninguna de las partes, el giro radical de los acontecimientos políticos a nivel internacional (el desmoronamiento súbito del bloque del Este) y regional (la ocupación militar de Panamá, la derrota electoral de los sandinistas en febrero de 1990 y la presencia de gobiernos conservadores en todos los países del istmo), y el cambio del paisaje político en el seno de El Salvador (la amplia victoria electoral de ARENA en marzo de 1988 y la elección, un año después, de su candidato como presidente de la República, junto con la ofensiva del FMLN sobre San Salvador en noviembre de 1989 - donde la organización guerrillera mostró su capacidad para continuar la contienda $\rightarrow$ mostraron la vía negociadora como el único medio eficaz para resolver la larga contienda.

El desenlace de un largo conflicto, en el caso salvadoreño, no fue fruto de ninguna victoria militar ni de ningún acontecimiento heroico, sino del cansancio y del hastío de las partes. Es necesario observar, sin embargo, que el 
tiempo corría en contra de la opción insurgente - las magras perspectivas en cuanto a futuras fuentes de financiamiento y provisión en un marco internacional hostil, y la progresiva dificultad de reclutar efectivos en un paisaje dominado por el agotamiento, cran factores que empujaban a los dirigentes del FMLN hacia soluciones de diálogo.

Los acuerdos de paz se firmaron en enero de 1992 en el castillo de Chapultepec (México). El cumplimiento de cada uno de los puntos establecidos es una tarea esencial para que se desarrolle un marco político que integre aquellos colectivos políticos que durante más de una década intentaron resolver sus pleitos por medio de la violencia. Con todo, el mantenimiento de instituciones que tuvieron gran centralidad durante el régimen anterior (las fuerzas armadas, el sistema judicial, etc.) y la persistencia de las profundas desigualdades son una flaca garantía para la viabilidad de un sistema llamado democrático.

\section{A modo de conclusión}

Los largos y traumáticos conflictos que tuvieron lugar durante más de diez años descompusieron en buena parte los antiguos modelos políticos, la cucstión estriba, sin embargo, en si posteriormente se pudo —o se podrá- recomponer un nuevo orden. La intervención estadounidense en estos procesos nos dice mucho acerca de la incapacidad de recomponerlo.

Los Estados nacional-populares de América del Sur necesitaron casi cincuenta ańos para consolidar su proyecto. En Centroamérica una década donde las reformas y transformaciones sc enmarañaron con guerras civiles, agresiones y crisis económicas de alcance internacional, fue un flaco contexto donde consolidar un modelo más equilibrado y justo para la convivencia.

Posiblemente los nuevos regímenes civiles, con sus nuevas instituciones, posibiliten una progresiva integración de las élites políticas que anteriormente se enfrentaron en la arena militar. El establecimiento de pautas de consenso supondrá, sin duda, un primer paso hacia la democratización de la vida política. Con todo, la permanencia de buena parte de los cuerpos que sustentaron el antiguo régimen y la casi intacta estructura de propiedad, cuestionan la consolidación de un orden estable a largo plazo en El Salvador. Nicaragua, por otro lado, se debate en medio de una frágil estabilidad; la satisfacción de las demandas de colectivos sociales en un período de posguerra - donde emergen aún manifestaciones de violencia de carácter económico o reivindicativo-, en el marco de una economía desencajada, parece una dificil ecuación para el afianzamiento de una democracia.

Pareciera que la estabilidad de las democracias y su génesis fueran dos fenómenos diferentes, como si después de crear los espacios necesarios para llevarla a cabo, las "aptas" e eimaginativas" elites perdieran parte de sus capacidades para hacer frente a las adversidades de su consolidación y estabilidad (Agüero y Iorcal, 1993; Karl, 1990; Higley y Gunther, 1992). O quizás el estudio de estos proceso va más allá del análisis de transiciones hacia ciertos regímenes 
(Levine, 1988). La permanencia de buena parte de los factores que quince años antes hicieron estallar la crisis en la región, las reducidas perspectivas de crecimiento económico de unas pequeñas economías tradicionalmente agroexportadoras, y la desaparición del istmo en la agenda política de la Administración de los EE UU en un contexto unipolar, hace recordarnos la ya recurrente frase de "nuevo vino en viejas botas?" (Remmer, 1991). La integración de estos elementos seguramente no sólo resultarían útiles para entender los actuales procesos de "democratización", sino también la naturaleza de las "democracias" resultantes (Smith, 1991; Whitehead, 1992).

\section{Bibliografia}

ACÚERO, F; TORCAL, M. (1993). "Élites, factores estructurales y democratización". Revista de Estudios Politicos, núm. 80, p. 329-350.

Baloyra, E. (1982). El Salvador in Transition. North Carolina Universiry Press. Chapell Hill.

- (1983). "Reactionary Despotism in Central America". Journal of Latin American Studies, núm. 15, p. 295-319.

BarRY, D.; Vergara, R.; Castro, R. (1987). Centroamérica: la Guerra de Baja Intensidad. San José: CRIES-DEI.

BARRY, G.; ROCAMORA, J. (1992). "Low intensity democracy". Third World Quartely, Vol. 13, 3, p. 501-523.

BENDANA, A. (1991). La guerra campesina. Managua: CRIES.

BERNTZEN, E. (1993). «A qualiative appoach to democratization in Central America". Ponencia presentada en ECPR Joint Sessions. Leiden.

BI.ACK, G. (1981). Triumph of the People: The Sandinista Revolution in Nicaragua. Londres: Zed Press.

Boort, J.A. (1991). "Socioeconomic and Political Roots of Narional Revolts in Central America». Latin American Research Review, núm. 26, p. 34-35.

BULMER-THOMAS, V. (1987). The Political Economy of Central America since 1920. Cambridge: Cambridge University Press.

CARMAGNANI, M. (1982). La grande illusione delle oligarchie. Stato e societa in America Latina (1850-1930). Turin: Loescher.

Cavarozzi, M. (1991). "Más allá de las transiciones a la democracia en America Latinaw. Revista de Estudios Políticos, núm. 74, p. 85-11 I.

Cardenal, A.S. (1993). Actores y cambio politico en El Salvador. Tesina de M.A. en Ciencias Políticas por la Universitat Autònoma de Barcelona. Barcelona.

Chiristian, S. (1986). Nicaragua. Revolución en la familia. Barcelona: Editorial Planeta.

COLLIER, D. (ed.). (1979). The New Authoritarianism in Latin America. Princeton: Princeton University Press.

Col.LIER, B.C.; COL.JIF, D. (1991). Shaping the Political Arena. Princeton: Princeton University Press.

DAllL, R. (1971). Polyarchy, Participation and Opposition. New Haven: Yale University Press.

DEUSTCH, K. (1961). "Implicaciones de la movilización social para la política del desarrollon. En Blondel y otros. El gobierno: estudios comparados. Madrid: Alanza, 1981. 
DORE, E.; WeEKS, J. (1992), "The Red and the Black. The Sandinistas and the Nicaraguan Revolutionm. Londres: Institute of Latin American Studies.

Drago, T. (1988). Centroamérica, una paz posible. Madrid: País-Aguilar.

DUNKERLEY, J. (1982). The Long War: Dictatorship and Revolution in El Salvador. Londres: Junction Books.

- (1990). Power in the Isthmus. Londres: Verso.

FLORA, J.L.; TORRES-RIVAS, E. (1989). «Sociology of Developing Societies: Historical Bases of Insurgency in Central Americam. En FLORA, J.L.; TORRES-RIVAS, E. (eds.). Central America. Londres: Macmillan Education.

GetTleman y ortos. (1981). El Salvador. Central America in the New Cold War. Nueva York: Grove Press, Inc.

GONZÁLEZ, S. (1992). El sandinismo: Análiisi de un cambio de régimen. Madrid: Universidad Complutense de Madrid, Phd Thesis.

HigleY, J.; GUNTHER, R. (eds.) (1992). Elites and democratization consolidation: Latin America and southern Europe. Cambridge: Cambridge University Press.

Huntington, S. (1984). "Will more Countries Be Democratic?». Political Science Quartely 1986, núm. 2, p. 193-218.

IBARRA, P. (1991). Centroamérica. Conflicto y negociacionn. Madrid: Libros de la catarata-HEGOA.

Karl, T.L. (1986). "Democracy by Design: the Christian Democratic Party in El Salvadorn. En, Di PALMA, G.; WhrTeHEAD, L. (eds.). The Central American Impasse. Londres.

- (1989). "The Christian Democratic Party and the Prospectsfor Democratization in El Salvadon, En, Flora, J.L.; TorRes-RIVAS, E. (eds.). Central America. Londres: Macmillan Education.

- (1990), "Dilemmas of Democratization in Latin America». Comparative Politics, núm. 23, p. 1-30.

LAFEBER, W. (1983). Inevitable Revolutions: The United States in Central America. Nueva York: W.W. Norton.

Levine, D. (1988). "Paradigm Lost: Dependency to Democracy». World Politics, núm. 40.

LiNZ, J.J. (1975), "Totalitarian and Authoritarian Regimes". En GreENSTEIN, F; POLSBY, N. (eds.). Handbook of Political Science, Vol. 3. Reading: Addison Wesley.

MARTí, S. (1994a). «El FSLN, del poder revolucionario a la oposición parlamentaria». En KRENNERICH; SANAHUjA (eds.). Nicaragua: el largo camino de la democratización. (forthcoming).

- (1994b). «Las fuerzas políticas no sandinistas, de la atomización a la UNOm. En KRENNERICH; SANAHUJA (eds.). Nicaragua: el largo camino de la democratización. (forthcoming).

MaRTínEZ, A. (1994). Del Enfrentamiento a la negociación. Factores que determinaron el fin de la Guerra Civil por medio de la Negociación. Tesina de M.A. en Ciencias Políticas por la Universitat Autònoma de Barcelona. Barcelona.

MCCuintock, M. (1985). The American Connection. State, Terror and Popular Resistance in El Salvador. Vol.1. Londres: Zed Books.

MONTGOMERY, T.S. (1982). Revolution in El Salvador: Origins and Evolution. Bouider: Westview Press.

MOORE, Jr.B. (1966). Social Origines of Dictatorship and Democracy. Lord in the Making of the Modern World. Boston: Beacon Press.

NúNEZ, y otros. (ed.) (1991). La guerra en Nicaragua. Managua: CIPRES-NORAD. 
PALMA, G. DI; WHitehEAD, L. (eds.) (1986). The Central American Impasse. Londres. REMMFR,K.L. (1991). "New Wine or Old Botzleneks? The Study of Latin American Democracies". Comparative Politics, julto 1991, p. 479-495.

ROBINSON, W.I.; NORSWORTHY, K. (1987). David and Goliath. Washington war against Nicaragua. Nueva York: Zed Books.

ROKKAN, S. (1975). "Dimensions of State Formation and Nation-Building: A Possible Paradigm for Research on Variations vithin Europe». En TiLly (ed.) The Formation of National States in Western Europe. Princeton: Princeton University Press.

SCHUl2, D.E; GRAHAM, D.H. (eds.) (1984). Revolution and Counterrevolution in Central America and the Caribbean. Boulder: Westview Press.

SERRA, L. (1988). "Organizaciones populares: entre las bases y el poder". Pensamiento Propio, núm. 30.

SMrTh, P.H. (1991). "Cisis and Demkocracy in Latin America". World Politics, núm. I2, p. 608-634.

SOlORZANO, M. (1983). "Centroamérica: Democracias de fachada". Polémica, núm. 12. Tinoco, V.H. (1989). Conflicto y paz en Centroamérica. México: Editorial Mestiza.

TORRES-RIVAS, E. (1983). Crisis de poder en Centroamérica. San José: EDUCA.

- (1990). "La recomposición del orden: elecciones en centroamética». Reis, núm. 50.

Touraine, A. (1989). America Latina. Politica y Sociedad. Madrid: Espasa Calpe.

W.AA. (1987). La transición Dificil. La autodeterminación de los pequeños patses periféricos. Managua: Editorial Vanguardia.

VILAS, C.M. (1984). Perfiles de la Revolución sandinista. La Habana: Ediciones Casa de las Américas.

- (1990). "Especulaciones sobre una sorpresa: las elecciones en Nicaragua». Revista de Ciencias Sociales Desarrollo Económico, núm. 118, p. 250-276. Buenos Aires.

- (1991a). "Facrores exógenos y elecciones en la democratización de Nicaragua". Documento presentado en LASA, XVI International Congress.

- (1991b). Una patria para todos: Revolución, Desarrollo y Democracia en Nicaragua. Ponencia presentada en en el Seminario «La democracia en America Latina: Actualidad y perspectivas", Madrid.

WALKER, T.H. (ed.) (1991). Revolution \& Cuonterrevolution in Nicaragua. Boulder: Westriew Press.

WaLter, K. (1993). The Somocista Regime. North Carolina University Press, ChapeI Hill.

WEEKS, J. (1986), "Land, Labour and Desporism in Central America». En PALMA, G. DI y WhiTeHEAD, (eds.). Central American Impasse. Nueva York: St. Martin's Press.

WHITEHEAD, L. (1992). "The Alternatives to "Liberal Democracy": on a Latin American Prespective». Political Studies, XL, Special Issue, p. 146-159.

WICKHAM-CROWLEY, T.P. (1993). Guerrillas and Revolution in Latin America: $A$ comparative study of Insurgents and Regimes since 1956. New Jersey: Princeton University Press. 